\title{
Dynamic Force Spectroscopy to Probe Adhesion Strength of Living Cells
}

\author{
K. Prechtel, ${ }^{1}$ A. R. Bausch, ${ }^{1}$ V. Marchi-Artzner, ${ }^{2}$ M. Kantlehner, ${ }^{3}$ H. Kessler, ${ }^{3}$ and R. Merkel ${ }^{4}$ \\ ${ }^{1}$ Lehrstuhl für Biophysik, E22, Technische Universität München, D-85747 Garching, Germany \\ ${ }^{2}$ Laboratoire de Chimie des Interactions Moleculaires UPR 285, College de France, \\ 11 Place Marcelin Berthelot, 75231 Paris Cedex 05, France \\ ${ }^{3}$ Lehrstuhl 2 für Organische Chemie, Technische Universität München, D-85747 Garching, Germany \\ ${ }^{4}$ Institut für Schichten und Grenzflächen, ISG 4, Forschungszentrum Jülich GmbH, 52425 Jülich, Germany
}

(Received 3 September 2001; published 20 June 2002)

\begin{abstract}
We studied the mechanical strength of the adhesion of living cells to model membranes. The latter contained a RGD lipopeptide which is a high affinity binding site for a cell adhesion molecule (integrin $\alpha_{V} \beta_{3}$ ). Cells adhered specifically to the vesicles. We used micropipette aspiration for breaking this adhesion with well defined forces. Systematic variation of the rate of force application revealed pronounced kinetic effects. The dependence of the detachment forces on the loading rate was well described by a power law (exponent $\approx 0.4$ ), in agreement with recent theoretical work.
\end{abstract}

DOI: 10.1103/PhysRevLett.89.028101

PACS numbers: 87.16.Dg, 87.15.By, 87.15.Kg

Cell-matrix adhesion plays an important role in many physiologically important processes such as tumor development and growth or wound healing [1]. Each cell bears a multitude of different cell adhesion molecules (CAMs) on its surface. A cell adheres to a given matrix only if some of its CAMs recognize a complementary part on the matrix. The chemical bonds between complementary CAMs exhibit comparatively low bond energies $(\sim 0.5 \mathrm{eV})$, yet astonishing specificity, i.e., the ability to distinguish between very closely related molecules such as blood group antigens. Therefore these bonds are aptly called "specific bonds." To achieve stable cell attachments nature compensates the low energies of these single bonds by using many of them in parallel. The mechanical strength of local cell adhesion is a key issue for many physiological processes as witnessed by the example of cell locomotion where bonds must be formed and broken in a tightly controlled process.

In recent studies on single specific bonds it was shown that the dissociation rate of such bonds is dramatically increased upon force application [2]. However, the implications of this finding for typical physiological situations where many molecular bonds share the overall mechanical load remains to be explored. In such a situation one single broken bond may be reestablished because the other bonds still hold the cells in place [3]. This implies that bond dissociation and failure of adhesion are no longer synonymous as is the case in experiments on single bonds. Thus we expect entirely new phenomena.

Experiments on enforced separation of adherent cells are very difficult to interpret because usually more than one type of CAMs participates, the cell surface is highly corrugated, and the mechanical properties of cells vary drastically as function of time and location within the cell [4]. These complications make it close to impossible to determine the distribution of force between different bonds and the time course of force application. In this Letter we introduce a novel approach that allows quantitative mea- surements of the mechanical strength of specific cellular adhesion mediated through multiple bonds, a situation most relevant for most physiological processes. The key step in this approach is replacing the matrix by a well defined functionalized model membrane, in our case a giant unilamellar vesicle (GUV). The latter was converted into a bioadhesion target by incorporation of a specially synthesized lipopeptide [5]. The peptide part of this molecule closely mimics a cell adhesion motif from vitronectin, a major structural protein of the extracellular matrix [6]. This peptide is recognized by a member of the integrin receptor family, $\alpha_{V} \beta_{3}$. This cell adhesion molecule is a receptor for vitronectin and is present at a density of $\approx 100$ molecules per $\mu \mathrm{m}^{2}$ on the surface of cultivated human endothelium cells [7]. Other CAMs exhibit very poor binding to this specific peptide. This design of the experiment ensures that just one type of specific bonds is probed. Moreover, model membranes are homogeneous and their mechanical properties are very well known [8]. Therefore we could analyze the mechanical load on the adhesion zone via the deflection of the model membrane. Hence, the model membrane served two purposes at the same time: it presented cell adhesion molecules and acted as a soft spring for force measurements.

In our experiments we used endothelium cells from human umbilical cord. Cells were grown in endothelium cell medium containing $10 \%$ fetal calf serum on collagen beads. Unilamellar giant vesicles containing the lipopeptide [9] were injected into the measurement chamber on the stage of a light microscope. Collagen beads bearing cells were added. Experiments were performed in phosphate buffered saline at a temperature of $37^{\circ} \mathrm{C}$ to ensure physiological conditions for the cells [10]. In the actual experiments a collagen bead on which a single endothelial cell was adhered was picked up by a micropipette [11]. With the opposing pipette a vesicle was collected and gently brought into contact with an endothelium cell. All 
measurements were observed by video microscopy and evaluated by digital image processing.

Following the contact of cell and model membrane the contact zone grew within several minutes. This growth did not affect the vesicle shape. In the further course of the experiments cell and vesicle were mechanically separated by gently retracting the pipette holding the vesicle using a piezoelectric translation stage; cf. Fig. 1. This procedure resulted in substantial deformations of the vesicle indicating mechanically strong adhesion [13]. Failure of the intermembrane adhesion occurred suddenly, i.e., within one video frame $(\approx 40 \mathrm{msec})$. At the membrane tensions used here $(\geq 90 \mu \mathrm{N} / \mathrm{m})$ the overall shape of the vesicle is entirely dominated by surface tension [14]. Therefore we can calculate the mechanical force at failure and the rate of force application by numerical methods developed earlier [12]. As sketched in Fig. 2, a tensile force acts only at the perimeter of the adhesion zone, in a ring of finite width [15]. In the vast majority of experiments the cell shape remained unchanged during enforced membrane separation. Moreover, the adhesion zones appeared always homogeneous. Therefore we calculated the forces assuming the cell to be homogeneous, flat, and not deformable. The rate of force application was systematically varied from
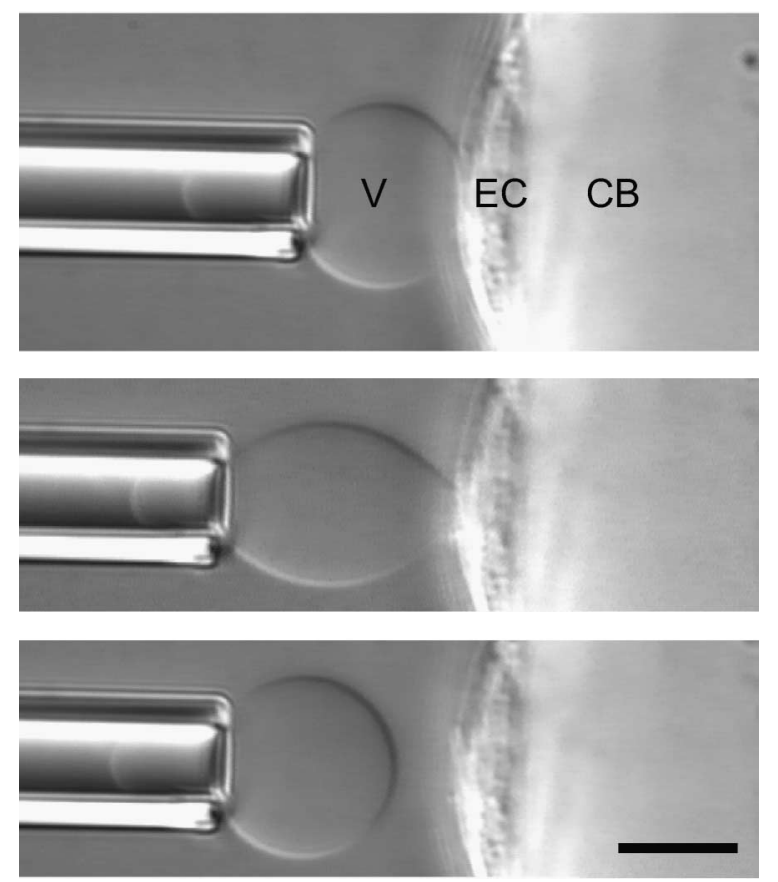

FIG. 1. A typical experiment. Top: A functionalized vesicle (V) in contact with an endothelium cell (EC) grown on a collagen bead $(\mathrm{CB})$. The vesicle membrane was tensed by pipette suction $(200 \mathrm{~Pa})$. Middle: The adhesion zone was loaded with a mechanical force of $4 \pm 1 \mathrm{nN}$. Because of this force the intermembrane adhesion broke instantaneously. Bottom: Immediately after the failure of adhesion. The rate of force application was $600 \pm 150 \mathrm{pN} / \mathrm{sec}$, which was determined from the retraction speed of the pipette $(0.5 \mu \mathrm{m} / \mathrm{sec})$ and the stiffness of the vesicle [12]. Scale bar, $10 \mu \mathrm{m}$.
$20 \mathrm{pN} / \mathrm{sec}$ to $4 \mathrm{nN} / \mathrm{sec}$ by changing the suction pressure and the retraction speed of the micropipette. The results of these experiments are displayed in Fig. 3. Obviously, the forces necessary to separate cell and vesicle are strongly correlated with the rate of force application. This was most convincingly demonstrated in experiments where we varied the suction pressure of the pipette from low to high and back during repeated adhesion tests employing the same cell and vesicle pair. The resulting yield forces followed exactly the changing loading rate. These data points cannot be distinguished from data collected on different cells at different rates. Thus our data clearly show that the enforced separation of a continuum of specific bonds is dominated by kinetic effects.

In our experiments very many molecules connect vesicle and cell simultaneously [17]. Thus the load on the individual bonds is rather low. The same holds for the adhesion between cells [1]. In such a situation binding and rebinding kinetics of the bonds is of great importance for the overall stability of the intercell adhesion [3]. As the lipopeptide is present in large excess over the integrin, the

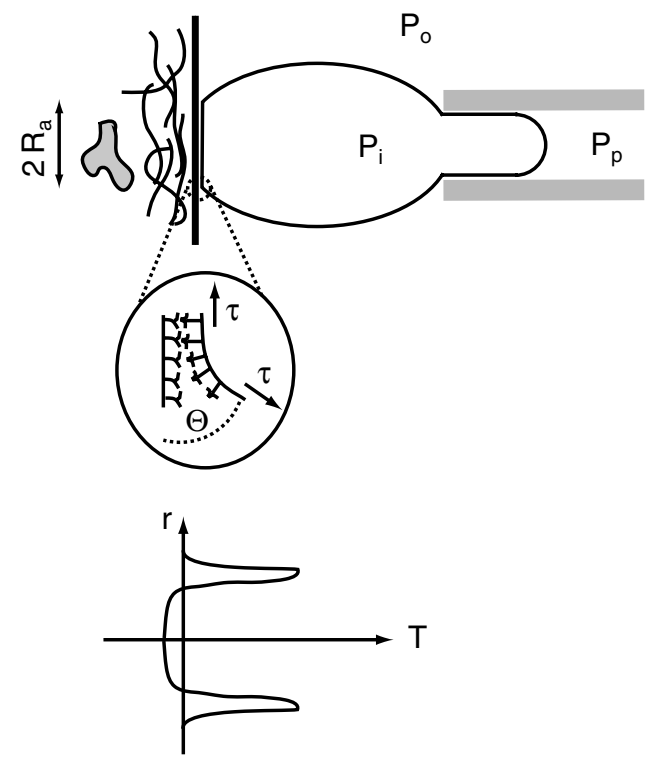

FIG. 2. Geometry of the adhesion test (top). Adhesion occurs within a circular region of radius $R_{a}$. The vesicle is held by micropipette aspiration; therefore its inner pressure, $P_{i}$, exceeds both the pressure within the pipette, $P_{p}$, and the pressure in the chamber, $P_{o}$. Thus the vesicle membrane is gently pressed against the cell (top left) in the interior of the adhesion zone. Tensile stress of the bonds arises solely at the rim of the adhesion zone where the membrane bends (inset). Because of the bending stiffness of the membrane, $\kappa$, bending occurs continuously within a bending zone of width $\sim \sqrt{\kappa / \tau}$, where $\tau$ denotes membrane tension. In the lower part of the figure the stress, $T$ (force/area), acting on the bonds is sketched. In the interior of the adhesion zone a compressive pressure $P_{i}-P_{o}$ acts; the peak of the tensile stress at the rim of the contact zone exhibits a width of $\sim \sqrt{\kappa / \tau}$ and an area of $\tau \sin \Theta$ where $\Theta$ is the macroscopic angle of membrane bending. 


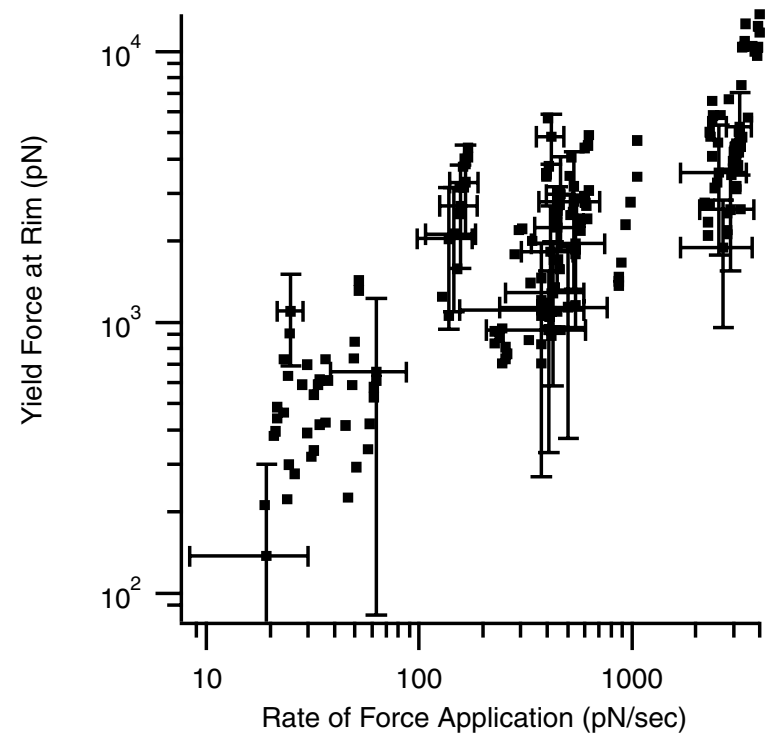

FIG. 3. The forces necessary to break the adhesion between cell and functionalized vesicle as a function of the rate of force application. Error bars are presented for $10 \%$ of all data points which were randomly chosen [16].

number of bonds, $N$, is governed by a pseudo first order kinetics.

$$
\frac{d N}{d t}=-k_{\mathrm{off}}(f) N+\left(N_{\mathrm{tot}}-N\right) k_{\mathrm{on}} .
$$

Here, $k_{\text {off }}$ is the force dependent dissociation rate of a single bond, $f$ is the force acting on it, $N_{\text {tot }}$ is the overall number of receptors in the adhesion zone, and $k_{\text {on }}$ is the pseudo first order association constant. In experiments on single bonds it was shown that $k_{\text {off }}$ usually follows an exponential dependence [2] $k_{\mathrm{off}}(f)=k_{0} \exp \left(\frac{f a}{k_{B} T}\right)$, where $a$ denotes the distance between bound state and transition state; i.e., it is a length on the order of $\sim 0.1 \mathrm{~nm}[2,18]$. Assuming this specific behavior of the force dependent dissociation rate and a geometry where many bonds experiencing equal forces are elastically connected to a rigid body, Seifert recently analyzed Eq. (1) [19] and found effective power laws for the dependence of the yield force per bond on the loading rate. Exponents ranged from 0.3 to 0.5 [20].

In our experiments tensile stress is limited to the bonds at the rim of the contact zone. Therefore we calculated the force density (tensile force per unit length) at the rim of the contact zone to remove the influence of different sized contact zones and to compare our data with the theoretical predictions of Seifert. As can be seen in Fig. 4 the data can be well described by a power law (exponent $0.36 \pm$ $0.1, \chi^{2} 1.8$; coinciding results were obtained using robust fitting algorithms).

In conclusion, we studied the adhesion between living cells and biofunctionalized model membranes. Cell adhesion was mediated by multiple bonds, which is the physiologically most relevant scenario. Employing the vesicle

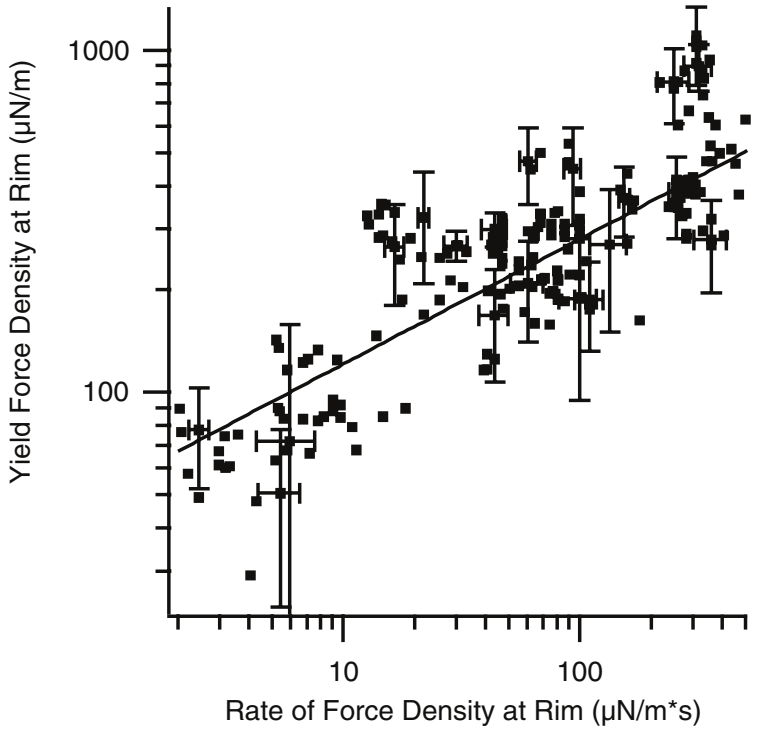

FIG. 4. The same data as Fig. 3 scaled by the circumference of the contact zone. The continuous line indicates the result of a fit to a general power law (exponent $0.36 \pm 0.1$ ).

as soft spring we were able to determine the dynamic yield forces with high accuracy. We find in our experiments that the mechanical adhesion strength depends very strongly on the duration of force application. Qualitatively, we find the same effect for multiple bonds that was found for single bonds, that is, lower yield forces for slower force application [2]. Quantitatively, the yield strength of multiple bonds depends on the force application rate according to a power law which is in good agreement with recent theoretical predictions [19]. In contrast, for single specific bonds a logarithmic dependence was reported. Thus, collective effects modify the scenario quantitatively but not qualitatively.

The results discussed above highlight the power of our approach which on one hand simplifies the very complex system, i.e., cell-matrix interaction, until quantitative conclusions can be drawn from the experiments. On the other hand, it conserves the salient features of the system, which are specific binding and multiple bonds to soft surfaces. The application of our approach to other types of cell adhesion bonds is evident.

Our observations are of profound importance for the physical understanding of many physiological processes such as tissue differentiation during embryonic development where adherent cell sheets are separated by a weak but steady osmotic pressure caused by the segregation of a biopolymer [21]. Any attempt to separate these cell sheets rapidly would result in high detachment forces with disastrous consequences for the cells. Yet, with such a steady pressure failure of adhesion occurs at negligible forces and is therefore well tolerated by the cells.

We thank Erich Sackmann for steady support and many helpful discussions. We had several enlightening discussions with Udo Seifert about his theory. Martin 
Aepfelbacher kindly supplied us with cells and Gabriele Chmel gave excellent technical assistance. This work was funded by the Deutsche Forschungsgemeinschaft (DFG) via the group Grant No. SFB266. A. B. thanks the DFG for support through the Emmy Noether program.

[1] B. Alberts, D. Bray, J. Lewis, M. Raff, K. Roberts, and J. D. Watson, Molecular Biology of the Cell (Garland Publishing, New York, 1994).

[2] R. Alon, D. A. Hammer, and T. A. Springer, Nature (London) 374, 539 (1995); K. D. Puri, S. Chen, and T. A. Springer, ibid. 392, 930 (1998); R. Merkel, P. Nassoy, A. Leung, K. Ritchie, and E. Evans, ibid. 397, 50 (1999); D. A. Simson, M. Strigl, M. Hohenadl, and R. Merkel, Phys. Rev. Lett. 83, 652 (1999); for a recent review, see R. Merkel, Phys. Rep. 346, 343 (2001).

[3] G. I. Bell, M. Dembo, and P. Bongrand, Biophys. J. 45, 1051 (1984); M. Dembo, D. C. Torney, K. Saxman, and D. Hammer, Proc. R. Soc. London B 234, 55 (1988); A. Tozeren, K.-L. Sung, and S. Chien, Biophys. J. 55, 479 (1989); C. Zhu, J. Biomech. 33, 23 (2000).

[4] A. R. Bausch, F. Ziemann, A. A. Boulbitch, K. Jacobson, and E. Sackmann, Biophys. J. 75, 2038 (1998).

[5] V. Marchi-Artzner, B. Lorz, U. Hellerer, M. Kantlehner, H. Kessler, and E. Sackmann, Chem. Eur. J. 7, 1095 (2001).

[6] The peptide contained the RGD (arginine-glycine-aspartic acid) motif; cf. M.D. Pierschbacher and E. Ruoslahti, Nature (London) 309, 30 (1984); J. W. Tamkun, D. W. DeSimone, D. Fonda, R. S. Patel, C. Buck, A. F. Horwitz, and R. O. Hynes, Cell 46, 271 (1986). We used the cyclic pentapeptide cyclo[Arg-Gly-Asp-D-Phe-Lys]. This peptide exhibits a defined molecular geometry, which greatly enhances the affinity, as shown by R. Haubner, R. Gratias, B. Diefenbach, S. L. Goodman, A. Jonczyk, and H. Kessler, J. Am. Chem. Soc. 118, 7461 (1996); M. Pfaff, K. Tangemann, B. Müller, M. Gurrath, G. Müller, H. Kessler, R. Timpl, and J. Engel, J. Biol. Chem. 269, 20233 (1994). The affinity of cyclic RGD peptides for different integrins was tested by measuring the peptide concentration necessary to reduce the amount of the respective integrins bound to vitronectin, their natural ligand, to $50 \%$. For the cyclic peptide used here this concentration was $\approx 2 \mathrm{nM}$ for the integrin $\alpha_{V} \beta_{3}$ and $\approx 50 \mu \mathrm{M}$ for $\alpha_{I I b} \beta_{3}$.

[7] E. F. Plow, J. C. Loftus, E. G. Levin, D. S. Fair, D. Dixon, J. Forsyth, and M.H. Ginsberg, Proc. Natl. Acad. Sci. U.S.A. 83, 6002 (1986); J. Lawler and R. O. Hynes, Blood 74, 2022 (1989).

[8] R. Lipowsky, Nature (London) 349, 475 (1991); M. Bloom, E. Evans, and O. G. Mouritsen, Q. Rev. Biophys. 24, 293 (1991).

[9] The vesicles were composed of di-elaidoyl-phosphatidylcholine, cholesterol, di-oleoyl-phosphatidyl-ethanolamine- poly(ethylen oxide) 2000 (a PEG lipid), and the lipopeptide in a ratio of 50:50:1:4. Using a 1:1 mixture of cholesterol and phospholipid as matrix ensures membrane fluidity and mechanical toughness. The polymer moiety of the PEG lipid forms a dense layer covering the membrane that efficiently suppresses nonspecific interactions between cell and vesicle. The concentration of the PEG lipid was selected because at much lower density its passivation potency is impaired and at much higher concentration it forms micellar phases. Identical results were obtained at a 100 times lower lipopeptide concentration; therefore we did not further vary its concentration.

[10] Cell viability was frequently checked after the experiments by the trypan blue test. In addition, no abnormal cell shapes (e.g., blebbing or rounding up) were observed. To ensure normal cell physiology the experiments were limited to durations of less than one hour.

[11] J. M. Mitchison and M. M. Swann, J. Exp. Biol. 31, 443 (1954); E. A. Evans, Methods Enzymol. 173, 3 (1989).

[12] E. A. Evans, Biophys. J. 31, 425 (1980); D. A. Simson, F. Ziemann, M. Strigl, and R. Merkel, ibid. 74, 2080 (1998).

[13] The specificity of this interaction was proven by three different tests: (i) experiments on cells that do not possess the integrin $\alpha_{V} \beta_{3}$ (fibroblasts), (ii) experiments with vesicles that did not contain the lipopeptide, and (iii) experiments in a buffer containing $2 \mathrm{mM}$ of the cyclic RGD peptide which saturated all binding sites. No adhesion was observed in any of these tests.

[14] E. Evans, in Handbook of Biological Physics, edited by R. Lipowsky and E. Sackmann (Elsevier Science, Amsterdam, 1995), Vol. 1.

[15] U. Seifert and R. Lipowsky, Phys. Rev. A 42, 4768 (1990).

[16] The total experimental error was calculated from the uncertainties of the individual input parameters for the calculation of the acting force, cf. [12], assuming that all contributions are uncorrelated and normally distributed. By far the largest contribution resulted from the determination of the radius of the adhesion zone because of the limited resolution of light microscopy. Variations between different cells are of much less importance.

[17] From the density of receptors $\left(\sim 100 \mu \mathrm{m}^{-2}\right)$ and the width of the bending zone $(\sim 0.2 \mu \mathrm{m}$; cf. Fig. 2$)$ we estimate that the line density of loaded bonds is $\sim 20 \mu \mathrm{m}^{-1}$. In our experiments the total number of loaded bonds ranged from 40 to 400 .

[18] G. I. Bell, Science 200, 618 (1978); E. Evans and K. Ritchie, Biophys. J. 72, 1541 (1997).

[19] U. Seifert, Phys. Rev. Lett. 84, 2750 (2000); Europhys. Lett. 58, 792 (2002).

[20] In Seifert's model the bonds withstand a finite force at vanishing loading rates. This cannot be seen in our data, most likely because the corresponding ultralow loading rates could not be reached.

[21] B. P. Toole, in Cell Biology of the Extracellular Matrix, edited by E. D. Haye (Plenum, New York, 1991). 